\title{
Alternative Voice Communication Device using Eye Blink Detection for People with Speech Disorders
}

\author{
Srividhya G, Murali S, A. Keerthana, Jaya Rubi
}

\begin{abstract}
Speech disorders is an inability to articulate sound due to medical ailments such as brain damage, stroke to paralysis and several other diseases. Research data suggests that about 15 million people in the world have partial or complete impairment of language expression due to brain damage, mostly from stroke. In this work a low-cost speech assistant device is proposed an alternate augmentative communication device for stroke or paralyzed patients. The eye blink sensor is used as the primary sensor for detection of eye blink. Based on the eye blink gestures the common words are displayed in the LCD and these words are also converted as voice output. This prototype will be an alternate communication aid for people with speech disorders.
\end{abstract}

Keywords : IR sensor, eye blink, voice.

\section{INTRODUCTION}

$S_{\text {peech disorders can affect the way of a person }}$ articulating sounds to form words. Speech disorders can be due to certain medical ailments starting from brain damage, stroke to paralysis and several other diseases. It can also result from motor damage during accidents which can leave a person completely ineffectual to communicate [2]. In paralysis the ability to control muscles movement is limited even around the eye muscles and blinks are the only way for the patient to communicate. In such communication the interface is often intrusive, which require special hardware or depend on active infrared sensors. Since speech impairment is not directly detrimental to the immediate health of the patient, it is often a neglected player in the development of medical device.

The different technologies used for implementing the communication between paralyzed patient and the people attending and caring for them are mouth actuated joysticks, actuated breathe puffing straws, tongue movement analysis, switch mounted near user's head, etc. These systems are costly to implement, increase stress on the patients and need skilled labor to setup and maintain the system for proper functioning. Hardly any devices have been developed that can address this issue in a patient friendly and cost-effective manner.

This work aims to design a simple and cost-effective voice

Revised Manuscript Received on November 25, 2019.

G.Srividhya, Assistant professor Department. Of Biomedical Engineering Vels Intitute of Science, Technology and Advanced Studies Higher educational institution in Chennai, Tamil Nadu

Murali;S, student in Vels Intitute of Science, Technology and Advanced Studies. Higher educational institution in Chennai, Tamil Nadu

A.Keerthana, Assistant Department, Professor in Biomedical Engineering VISTAS, Chennai, India. communication device for patients suffering from speech disorder using eye blink of the patient. Eye blink at regular intervals can be interpreted for forming words for communication. An efficient, real time blink detection algorithm can be used for almost any purpose like switching ON/OFF of appliances at home etc, [1].

\section{METHODOLOGY}

In this work a low-cost speech assistant device is proposed as an alternate augmentative communication device. The eye blink sensor is used as the primary sensor for detection of eye blink [5]. Eye blinking is one of the most common and convenient human activities that can be used as an input modality for a simple communication methodology $[3,6]$ and eye blinking can be an indicator for certain human behaviors such as patients with Parkinson's disease have altered eye blink characteristics [4]. Based on the eye blink gestures the common words are displayed in the LCD and these words are also converted as voice output. This prototype will be an alternate communication aid for patients with speech disorders.

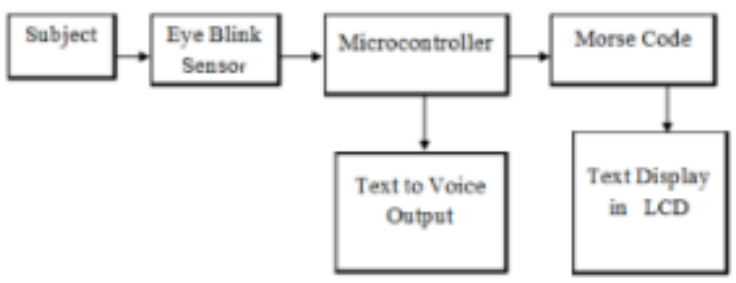

Figure 1 Block Diagram

The above block diagram describes the steps involved in the design of this augmentative device. IR led and sensor is used for detecting eye blink [1]. The voltage values during eye closed and eye open is fed to a microcontroller for analysis of the rate eye blink and the duration of eye blink [8,9]. These eye movement data are converted to the corresponding alphabet using Morse code. These are displayed in an LCD screen and the text is converted to voice through voice converter module.

\section{A. IR Sensor Module:}

IR sensor used in this work is QRD1114 which is a half LED and half phototransistor device. It can be used to sense objects in close proximity or even detect the difference between dark and light colored surfaces. The IR sensor used does not cause any harm or irritation to the eyes and has minimized or no effects on the eye tissues. The QRD1114 is a 4-pin device. Two pins break out the infrared 
LED, and the other two pins break out the collector and emitter pins of the phototransistor.

\section{B. LCD and Audio Amplifier module:}

$6 \times$ 2-character LCD display is used to display the words. LM386 is a low voltage audio amplifier. This audio amplifier is used to give voice output of the text displayed.

\section{Interfacing with Microcontroller}

Among the several available microcontroller modules Arduino microcontroller has been chosen for this work as it is user friendly, flexible and very economical. The IR sensor, LCD display and the audio amplifier are interfaced with the Arduino Microcontroller.

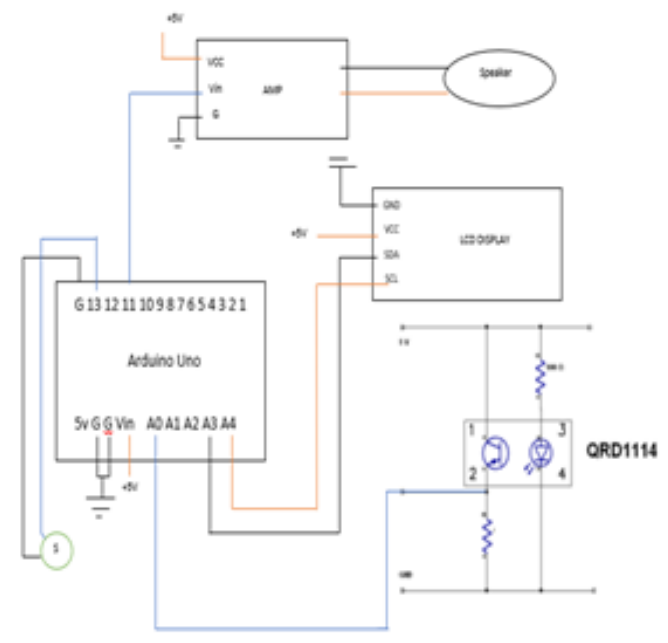

Figure 2: Circuit Diagram

\section{Morse Code Interpretation}

Morse code is a character encoding method used in telecommunication where the text characters are encoded as standardized sequences of two different signal durations. These signal durations are called as dots and dashes [12]. The duration of dot signal is the basic unit of time measurement and dash is thrice the duration of dot [11].
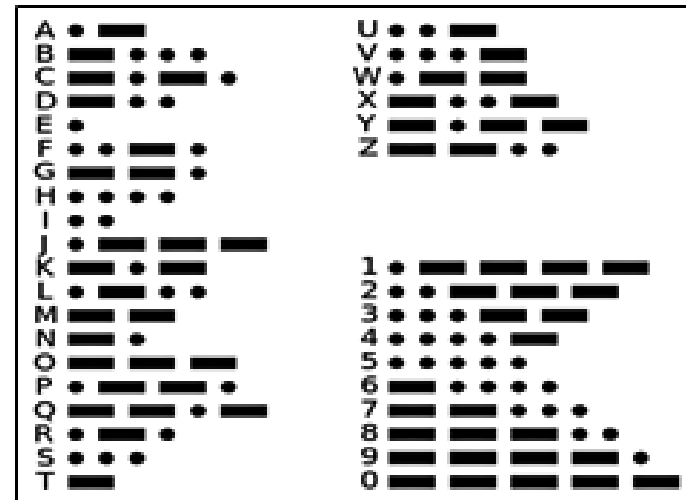

Figure 3: Morse Code

\section{PROCEDURE}

The IR sensor value differs for eye closed and open. This variation is marked as corresponding dot and dash of the morse code. Initially, training is given to the subject to do eye blinks at regular interval. A voltage threshold is set to differentiate the status of eye.
Since dash is three times duration of dot, each and every dot or dash within a character is followed by a gap or space equal to the duration of dot. This pattern is coded in Arduino microcontroller board to the corresponding alphabetical character or number.

\section{RESULT AND DISCUSSION}

The IR Sensor senses the amount of reflected light from the eye and sends the signal to the Arduino. as the level reflection varies significantly between eye close and eye open, the voltage values are recorded to fix a threshold for eye blink. Table 1 shows the Eye open and close values.

Table I Eye open and Eye Closed IR sensor values

\begin{tabular}{|c|c|c|}
\hline S.No & Eye open & Eye close \\
\hline 1 & 56 & 283 \\
\hline 2 & 47 & 282 \\
\hline 3 & 57 & 304 \\
\hline 4 & 56 & 309 \\
\hline 5 & 60 & 264 \\
\hline
\end{tabular}

In this work, short blink of the person corresponds to a dot and long blink to a dash of a Morse code. The code for the alphabet $\mathrm{I}$ is a dot and a dot and for $\mathrm{H}$ is a dot and a dot and a dot and a dot. Figure 3 shows the LCD display of the word "HI".

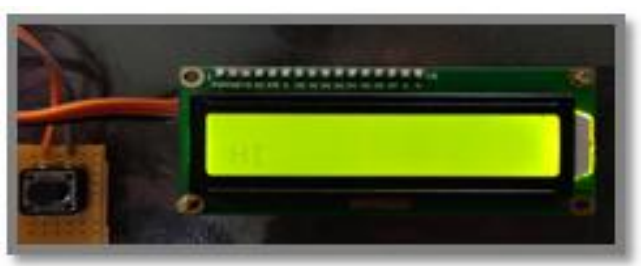

Figure 4: LCD display of word "HI"

This pattern is fed to the audio amplifier and voice output of the words is obtained. Initially, the subject was trained with more interval of eye blink which is interpreted as Morse code by Arduino. The communication through eye blink was smooth and quick and was able to do with a lot of spontaneity as our regular communication method. Once certain familiarity has been achieved the code can work fast. While entering spontaneously it took an average duration of 5 seconds to communicate an alphabet. But this duration varies from one alphabet to another as some had more dots (short blinks) while some had more dashes (longer blinks).

\section{CONCLUSION}

In this work, a prototype of text to voice communication device with eye blink detection technique for person with speech disorders is designed. The variation of IR sensor value during eye blink is taken as threshold and the interval of variation is used as Morse code that is universally accepted code of communication. After every eye blink the microcontroller detects the change in the interval and stores as corresponding Morse code. This is detected as particular alphabet and it is displayed onto the LCD screen. This alphabet or command is converted to the voice output.

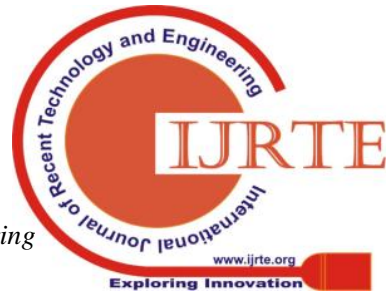




\section{REFERENCES}

1. Atish Udayashankar, Amit R. Kowshik, S. Chandramouli, H.S. Prashanth-'Assistance for the Paralyzed Using Eye Blink Detection', 2012 Fourth International Conference on Digital Home,Year:2012.

2. Kingshuk Mukherjee, Debdatta Chatterjee," Augmentative and Alternative Communication Device Based on Eye-Blink Detection and Conversion to Morse-Code to Aid Paralyzed Individuals",2015 International Conference on Communication, Information \& Computing Technology (ICCICT),year:2015.

3. Youngwook Kim," Detection of Eye Blinking Using Doppler Sensor with Principal Component Analysis", IEEE Antennas and wireless propagation letter,year;2015,volume:14

4. Woon-Hee Lee, Jong-Mo Seo*, Jeong-Min Hwang," The Analysis of Eye Blinking Pattern Using High-Frame-Rate Camera”2017 $39^{\text {th }}$ annual international conference of IEEE engineering in medicine and biology society(EMBC),Year:2017.

5. Mustafa El-Halabi, Rayan Haidar, Rami El Kadri, Chadi Lahoud," Eye-Blinks Communication Vehicle: A Prototype" 2017 Fourth International Conference on Advances in Biomedical Engineering (ICABME),year:2017.

6. MadhumantiMaiti, Tanaya Banerjee,"An Innovative Prototype to Prevent Accidents Using Eye Blink Sensors and Accelerometer ADXL330",2015 international conference and workshop on computing and communication(IEMCON), Year;2015.

7. Koichi Takahashi1, Yasue Mitsukura1," Eye Blink Detection Using Monocular System and its Applications", The 21st IEEE International Symposium on Robot and Human Interactive Communication, year: 2012.

8. Fabio Lo Castro "Class I infrared eye blinking detector”, Volume-48, Issue 2, 3 December 2008, Pages 388-394, DOI10.1016/j.sna.2008.09.005, Elsevier.

9. Philip Graybill, Mehdi Kiani," Eyelid Drive System: An Assistive Technology Employing Inductive Sensing of Eyelid Movement", IEEE Transactions on Biomedical Circuits and Systems, year:2018.

10. Marc Lalonde, David Byrns, Langis Gagnon, Normand Teasdale†, Denis Laurendeau," Real-time eye blink detection with GPU-based SIFT tracking", year:2007.

11. Ricky Li, Minh Nguyen, Wei Qi Yan, "Morse Codes Enter Using Finger Gesture Recognition", Year:2017.

12. L. R..Sapaico, and M. Sato, "Analysis of vision-based Text Entry using morse code generated by tongue gestures," In International Conference on Human System Interactions, year:2011.

\section{AUTHORS PROFILE}

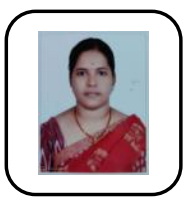

book chapters.

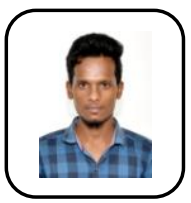

Murali;S is student in Vels Intitute of Science, Technology and Advanced Studies. Presented a paper at National Conference held at Vels University on March 2019.

is currently associated with Vels Intitute of Science, Technology and Advanced Studies as Assistant professor in Biomedical Engineering Department. She has a research experience in Anna Univeristy. She is interested in Medical Image processing. She wrote research papers which is published in reputed journals. She also wrote two 